\title{
Hyperdeterminants on semilattices
}

\author{
Jean-Gabriel Luque *
}

November 22, 2006

\begin{abstract}
We compute hyperdeterminants of hypermatrices whose indices belongs in a meet-semilattice and whose entries depend only of the greatest lower bound of the indices. One shows that an elementary expansion of such a polynomial allows to generalize a theorem of Lindström to higher-dimensional determinants. And we gave as an application generalizations of some results due to Lehmer, Li and Haukkanen.
\end{abstract}

keywords:Hyperdeterminants, meet semilattices, GCD-matrices, multiplicative functions.

AMS: 15A15, 15A69, 06A12.

\section{Introduction}

Since the end of the nineteen century, it is known that some determinants, with entries depending only of the gcd of the indices, factorize. Readers interested in the story of the problem can refer to [13] and [2]. In 1876, Smith [15] evaluated the determinant of a GCD matrix whose entries belong to a factor closed set (i.e., a set which contains all the factors of its elements) as a product of Euler's totient. The interest of this computation lies in its links with arithmetic functions [1] and in particular multiplicative functions (see [7, 8] for interesting remarks about the last notion). During the last century, many generalizations of Smith's theorem have been investigated. One of the

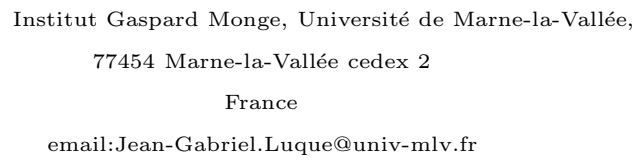

email:Jean-Gabriel.Luque@univ-mlv.fr 
ways to extend his result consists in changing the set of the indices of the matrices. In 1990, Li [10] gave the value of GCD determinant for an arbitrary set of indices. Beslin and Ligh [3] shown that such a determinant factorizes when the indices belongs to a gcd-closed set (i.e., a set which contains the gcd of any pairs of its elements) as a product of certain functions evaluated in terms of Euler's totient. The fact that these determinants factorize can be seen as a corollary of a very elegant theorem due to Lindström [11] which evaluated the determinant of the GCD-matrix whose indices are chosen in a meet semilattice, i.e., a poset such that each pair admits a greatest lower bound. Another way to generalize Smith's result consists in computing multidimensional analogous. In 1930 Lehmer gave [9] the first multi-indexed version of Smith's determinant. Other related computation are collected in [16, 17]. More recently, Haukkanen [12] generalized the results of Beslin and Ligh [3] and Li [10]) to hyperdeterminants.

We will see in Section 2 that the main trick for computing these multidimensional determinants consists in expanding it as a sum of (classical 2-way) determinants. In the aim to highlight this method, we apply it to a more general object $\operatorname{Det}_{\mathfrak{F}}$.

In Section 3, we recall shortly a classical technic and give a slight generalization of Lindström's Theorem. As a consequence, we give a multidimensional analogue of Lindström's Theorem.

In Section 1, one investigates minors of meet hypermatrices and generalizes two theorems due to Haukkanen [12].

\section{$2 \quad$ Hyperdeterminants and $\mathfrak{F}$-determinants}

The question of extending the notion of determinant to higher dimensional arrays has been raised by Cayley [5, 6] few after he introduced the modern notation as square arrays [1. The simplest generalization is defined for a $k$ th order tensor on an $n$-dimensional space $M=\left(M_{i_{1}, \cdots, i_{k}}\right)_{1 \leq i_{1}, \cdots, i_{k} \leq n}$ by the alternated sum

$$
\operatorname{Det} M=\frac{1}{n !} \sum_{\sigma=\left(\sigma_{1}, \cdots, \sigma_{k}\right) \in \mathfrak{S}_{n}^{k}} \operatorname{sign}(\sigma) M^{\sigma},
$$

where $\operatorname{sign}(\sigma)=\operatorname{sign}\left(\sigma_{1}\right) \cdots \operatorname{sign}\left(\sigma_{k}\right), M^{\sigma}=M_{\sigma_{1}(1) \ldots \sigma_{k}(1)} \cdots M_{\sigma_{1}(n) \ldots \sigma_{k}(n)}$ and $\mathfrak{S}_{n}$ is the symmetric group. A straightforward computation gives $\operatorname{Det} M=0$ 
if $k$ is odd.

For any $k$ (even if $k$ is odd), one defines the polynomial

$$
\operatorname{Det}_{1} M=\sum_{\sigma=\left(\operatorname{Id}, \sigma_{2}, \cdots, \sigma_{k}\right) \in \mathfrak{S}_{n}^{k}} \operatorname{sign}(\sigma) M^{\sigma} .
$$

When $k$ is even the two notions coincide but for $k$ odd, only Det $_{1}$ does not vanish. This is a special case of the "less-than-full-sign" determinant theory due to Rice [14].

Let us denote by $\mathfrak{F}$ a map from $\mathfrak{S}_{n}^{k-2}$ to a commutative ring. One defines a more general object, which will be called $\mathfrak{F}$-determinant of $M$ by

$$
\operatorname{Det}_{\mathfrak{F}}(M)=\sum_{\sigma=\left(\sigma_{2}, \cdots, \sigma_{k}\right) \in \mathfrak{S}_{n}^{k-1}} \operatorname{sign}\left(\sigma_{2}\right) \mathfrak{F}\left(\sigma_{3}, \ldots, \sigma_{k}\right) \prod_{i} M_{i \sigma_{2}(i) \ldots \sigma_{k}(i)} .
$$

It exists an elementary identity which consists in expanding the $\mathfrak{F}$-determinant as a sum of $(n !)^{k-2}$ classical (2-way) determinants.

Lemma 2.1 (Determinantal expansion)

One has

$$
\operatorname{Det}_{\mathfrak{F}} M=\sum_{\sigma_{3}, \ldots, \sigma_{k}} \mathfrak{F}\left(\sigma_{3}, \ldots, \sigma_{k}\right) \operatorname{det}\left(M^{\sigma_{3}, \ldots, \sigma_{k}}\right),
$$

where $M^{\sigma_{3}, \ldots, \sigma_{k}}$ denotes the $n \times n$ matrix such that $M_{i, j}^{\sigma_{3}, \ldots, \sigma_{k}}=M_{i, j, \sigma_{3}(i), \ldots, \sigma_{k}(i)}$. Proof - It suffices to remark that

$$
\operatorname{Det}_{\mathfrak{F}} M=\sum_{\sigma_{3}, \ldots, \sigma_{k}} \mathfrak{F}\left(\sigma_{3}, \ldots, \sigma_{k}\right) \sum_{\sigma_{2}} \operatorname{sign}\left(\sigma_{2}\right) \prod_{i} M_{i \sigma_{2}(i) \sigma_{3}(i) \cdots \sigma_{k}(i)}
$$

One of the most important property of hyperdeterminants is the invariance under the action of $k$ copies of the special linear group. It is a very classical result which can be recover as a straightforward consequence of the following proposition.

Proposition 2.2 The polynomial $\operatorname{Det}_{\mathfrak{F}} M$ is invariant under the action of linear group on $M$ in the following sense

$$
\operatorname{Det}_{\mathfrak{F}} g \cdot M=\operatorname{det} g \operatorname{Det}_{\mathfrak{F}} M,
$$

where

$$
g \cdot M=\left(\sum_{1 \leq j_{2} \leq n} g_{i_{2} j_{2}} M_{i_{1}, j_{2}, i_{3} \cdots, i_{k}}\right)_{1 \leq i_{1}, \cdots, i_{k} \leq n} .
$$


Proof - By applying Lemma 2.1 to g.M, one gets

$$
\begin{aligned}
\operatorname{Det}_{\mathfrak{F}} g \cdot M & =\sum_{\sigma_{3}, \ldots, \sigma_{k}} \mathfrak{F}\left(\sigma_{3}, \ldots, \sigma_{k}\right) \operatorname{det}\left(g \cdot M^{\sigma_{3}, \ldots, \sigma_{k}}\right) \\
& =\sum_{\sigma_{3}, \ldots, \sigma_{k}} \mathfrak{F}\left(\sigma_{3}, \ldots, \sigma_{k}\right) \operatorname{det} g \operatorname{det}\left(M^{\sigma_{3}, \ldots, \sigma_{k}}\right) \\
& =\operatorname{det} g \operatorname{Det}_{\mathfrak{F}} M .
\end{aligned}
$$

\section{Hyperdeterminants of meet hypermatrices}

\subsection{Meet semilattice}

Consider a partially ordered finite set $L$ so that every pairs $(x, y) \in L^{2}$ has a greatest lower bound denoted by $x \wedge y$. Such a poset is called a meet semilattice. One defines classically its $\zeta$ function by

$$
\zeta(x, y)= \begin{cases}1 & \text { if } x \leq y \\ 0 & \text { otherwise }\end{cases}
$$

Its Möbius function is the inverse of the zeta function and can be computed by the induction

$$
\mu(x, y)= \begin{cases}1 & \text { if } x=y \\ -\sum_{x \leq z<y} \mu(z, y) & \text { if } x<y \\ 0 & \text { in the other cases. }\end{cases}
$$

If $F$ and $f$ verify the equality

$$
F(x)=\sum_{y \leq x} f(y)=\sum_{y \in L} \zeta(y, x) f(y),
$$

then, one has

$$
f(x)=\sum_{y \in L} \mu(y, x) F(y)=(F \star \mu)(x)
$$

where the symbol $\star$ means the convolution product. 


\subsection{Lindström Theorem}

The factorization properties of the GCD determinants are the consequence of the semilattice structure of the integers with respect to divisibility and can be stated in a more general way. The manipulations of the identities (2) and (3) are the keys of the proof of Lindström's Theorem [11]. We recall its proof in a very slightly more general version.

For each $x \in L$, one considers a fixed element $z_{x} \leq x$. Let $F_{x}$ be a function from $L$ to $\mathbb{C}$ (or more generally to a commutative ring). Let $M$ be the matrix defined by

$$
M=\left(F_{x}\left(z_{x} \wedge y\right)\right)_{x, y \in L}
$$

Remark that it suffices to define $F_{x}(z)$ only when $z \leq x$. In particular, one can suppose that $F_{x}(z)=F(z, x)$ is an incidence function, i.e., $F(x, z)=0$ unless $x \leq z$. One has

$$
F_{x}\left(z_{x} \wedge y\right)=\sum_{z \in L} \zeta\left(z, z_{x}\right) \zeta(z, y) f_{x}(z)
$$

where $f_{x}(z)=\sum_{y \in L} \mu(y, z) F_{x}(y)$. Hence, $\operatorname{det} M$ factorizes as the product $\operatorname{det} M=\operatorname{det} \Phi \cdot \operatorname{det} Z$,

where $\Phi=\left(\zeta\left(y, z_{x}\right) f_{x}(y)\right)_{x, y \in L}$ and $Z=(\zeta(x, y))_{x, y \in L}$. As $\Phi$ and $Z$ are triangular, $\operatorname{det} Z=1$ and

$$
\operatorname{det} \Phi=\prod_{x} \zeta\left(x, z_{x}\right) f_{x}(x)= \begin{cases}\prod_{x} f_{x}(y) & \text { if } z_{x}=x \text { for each } x \\ 0 & \text { otherwise }\end{cases}
$$

Then one obtains Lindström's Theorem.

Theorem 3.1 (Lindström [11])

$$
\operatorname{det}\left(F_{x}\left(z_{x} \wedge y\right)\right)_{x, y \in L}= \begin{cases}\prod_{x} f_{x}(x) & \text { if } z_{x}=x \text { for each } x \\ 0 & \text { otherwise }\end{cases}
$$

Note that, the original Lindstöm 's Theorem deals with the case where $z_{x}=x$ for each $x$. Furthermore, equality (4) generalizes a lemma of Cesaro.

Lemma 3.2 (Cesaro)

Denote by $\operatorname{gcd}_{m}(n)=\operatorname{gcd}(m, n)$. One has,

$$
\left(\mu *\left(f \circ \operatorname{gcd}_{m}\right)\right)(n)= \begin{cases}(f * \mu)(n) & \text { if } m=n, \\ 0 & \text { otherwise, }\end{cases}
$$

where $*$ is the Dirichlet convolution and $\circ$ is the composition of functions. 


\subsection{Linström's Theorem for $\mathfrak{F}$-determinants}

Lindstöm's Theorem can be extended to $\mathfrak{F}$-determinants.

Theorem 3.3 (Lindström's theorem for $\mathfrak{F}$-determinants)

If $L=\left\{x_{1}, \ldots, x_{n}\right\}$ denotes a meet semilattice, one has

$\operatorname{Det}_{\mathfrak{F}}\left(F_{x_{i_{1}}}\left(z_{x_{i_{1}}} \wedge \cdots \wedge x_{i_{k}}\right)\right)= \begin{cases}\mathfrak{F}(\mathrm{Id}, \cdots, \mathrm{Id}) \prod_{x} f_{x}(x) & \text { if } z_{x}=x \text { for each } x \\ 0 & \text { otherwise }\end{cases}$

Proof - Lemma 2.1 gives

$$
\begin{aligned}
& \operatorname{Det}_{\mathfrak{F}}\left(F_{x_{1}}\left(z_{x_{1}} \wedge \cdots \wedge x_{k}\right)\right)_{x_{1}, \cdots, x_{k} \in L}= \\
& \sum_{\sigma_{3}, \ldots, \sigma_{k}} \mathfrak{F}\left(\sigma_{3}, \ldots, \sigma_{k}\right) \operatorname{det}\left(F_{x_{i}}\left(z_{x_{i}} \wedge x_{j} \wedge x_{\sigma_{3}(i)} \wedge \cdots \wedge x_{\sigma_{k}(i)}\right)_{i, j}\right.
\end{aligned}
$$

From Linström's Theorem (Theorem 3.1), one has

$$
\operatorname{det}\left(F_{x_{i}}\left(z_{x_{i}} \wedge x_{j} \wedge x_{\sigma_{3}(i)} \wedge \cdots \wedge x_{\sigma_{k}(i)}\right) \neq 0\right.
$$

if and only if for each $x_{i}$ one has

$$
z_{x_{i}} \wedge x_{\sigma_{3}(i)} \wedge \cdots \wedge x_{\sigma_{k}}(i)=x_{i} .
$$

Equivalently,

$$
z_{x_{i}} \wedge x_{\sigma_{3}(i)} \wedge \cdots \wedge x_{\sigma_{k}}(i) \geq x_{i},
$$

for eacu $i$. Hence, $\sigma_{3}=\cdots=\sigma_{k}=I d$ and $z_{x_{i}}=x_{i}$ for each $i$. The result follows.

Example 3.4 Consider the semilattice constituted with two elements $2 \geq 1$. The expansion of the $\mathfrak{F}$-determinant gives

$$
\begin{aligned}
\operatorname{Det}_{\mathfrak{F}}\left(F_{i}(i \wedge j \wedge k \wedge l)\right)= & \mathfrak{F}(12,12)\left|\begin{array}{ll}
F_{1}(1) & F_{1}(1) \\
F_{2}(1) & F_{2}(2)
\end{array}\right| \\
& +(\mathfrak{F}(12,21)+\mathfrak{F}(21,12)+\mathfrak{F}(21,21))\left|\begin{array}{ll}
F_{1}(1) & F_{1}(1) \\
F_{2}(1) & F_{2}(1)
\end{array}\right| \\
= & \mathfrak{F}(12,12) F_{1}(1)\left(F_{2}(2)-F_{2}(1)\right) \\
= & \mathfrak{F}(12,12) f_{1}(2) f_{2}(2)
\end{aligned}
$$




\section{Minors of meet Hypermatrices}

\subsection{Meet closed subsets}

Consider a meet closed subset $S$ of $L$ (i.e., a subset closed under the operation $\wedge$ ) and fix a linear extension $l=y_{1} \ldots y_{n}$ of $S$. Following the notations of [2], we denote by $x \unlhd y_{i}$ the relation $x \leq y_{i}$ and $x \not \leq y_{j}$ for each $j<i$. Consider a pair of functions $f$ and $F$ verifying

$$
F\left(y_{i}\right)=\sum_{\substack{x \leq y_{i} \\ x \in L}} f(x)
$$

and set $\hat{f}\left(y_{i}\right)=\sum_{x \unlhd y_{i}} f(x)$. One has the following lemma.

\section{Lemma 4.1}

$$
F\left(y_{i}\right)=\sum_{y_{k} \leq y_{i}} \hat{f}\left(y_{k}\right)
$$

Proof - Remarking that for each $x \in L$, it exists $i$ such that $x \unlhd y_{i}$ (it suffices to set $y_{i}=\min \left\{j \mid x \leq y_{j}\right\}$ and that $x \unlhd y_{i}$ and $x \unlhd y_{j}$ implies $i=j$, we have

$$
F\left(y_{i}\right)=\sum_{\substack{x \leq y_{i} \\ x \in L}} f(x)=\sum_{y_{k} \leq y_{i}} \sum_{x \unlhd y_{k}} f(x)=\sum_{y_{k} \leq y_{i}} \hat{f}\left(y_{k}\right) .
$$

Note that this identity appears in [2] (Theorem 4.1, p 7). Hence, using Theorem 3.3 and Lemma 4.1, one generalizes a result by Altinisik, Sagan and Tuglu ([2], Theorem $4.1 \mathrm{p} 7$ ).

\section{Corollary 4.2}

$\operatorname{Det}_{\mathfrak{F}}\left(F_{y_{i_{1}}}\left(y_{i_{1}} \wedge \cdots \wedge y_{i_{k}}\right)\right)=\mathfrak{F}(\mathrm{Id}, \cdots, \mathrm{Id}) \prod_{i=1}^{n}\left(\sum_{x_{1} \unlhd y_{i}} \sum_{x_{2} \in L} \mu\left(x_{1}, x_{2}\right) F_{y_{i}}\left(x_{2}\right)\right)$.

Example 4.3 Consider the semilattice L given by its Hasse diagram

$$
L=\begin{array}{lll}
4 & & 5 \\
\uparrow & \nearrow & \uparrow \\
2 & & 3 \\
\uparrow & \nearrow & \\
1 & &
\end{array}
$$


where $i \rightarrow j$ means $i \leq j$. The sublattice generated by 2 , 4 and 5 ,

$$
S=\uparrow_{2}^{4} \nearrow^{5}
$$

is meet closed and

$$
\begin{aligned}
& \hat{f}_{2}(2)=f_{2}(2)+f_{2}(1) \\
& \hat{f}_{4}(4)=f_{4}(4) \\
& \hat{f}_{5}(5)=f_{5}(5)+f_{5}(3) .
\end{aligned}
$$

Hence,

$$
\begin{aligned}
\operatorname{Det}_{\mathfrak{F}}\left(F_{i}(i \wedge j \wedge k)\right)_{i, j, k \in S}= & \mathfrak{F}(123)\left|\begin{array}{lll}
F_{2}(2) & F_{2}(2) & F_{2}(2) \\
F_{4}(2) & F_{4}(4) & F_{4}(2) \\
F_{5}(2) & F_{5}(2) & F_{5}(5)
\end{array}\right| \\
& +\mathfrak{F}(213)\left|\begin{array}{lll}
F_{2}(2) & F_{2}(2) & F_{2}(2) \\
F_{4}(2) & F_{4}(2) & F_{4}(2) \\
F_{5}(2) & F_{5}(2) & F_{5}(5)
\end{array}\right| \\
& +\mathfrak{F}(321)\left|\begin{array}{lll}
F_{2}(2) & F_{2}(2) & F_{2}(2) \\
F_{4}(2) & F_{4}(2) & F_{4}(2) \\
F_{5}(2) & F_{5}(2) & F_{5}(2)
\end{array}\right| \\
& +(\mathfrak{F}(132)+\mathfrak{F}(231)+\mathfrak{F}(312))\left|\begin{array}{lll}
F_{2}(2) & F_{2}(2) & F_{2}(2) \\
F_{4}(2) & F_{4}(2) & F_{4}(2) \\
F_{5}(2) & F_{5}(2) & F_{4}(2)
\end{array}\right|
\end{aligned}
$$

The permutation 123 is the only one having a non zero contribution in this sum. Hence,

$$
\begin{aligned}
\operatorname{Det}_{\mathfrak{F}}\left(F_{i}(i \wedge j \wedge k)\right)_{i, j, k \in S} & =\mathfrak{F}(123) F_{2}(2)\left(F_{4}(4)-F_{4}(2)\right)\left(F_{5}(5)-F_{5}(2)\right) \\
& =\mathfrak{F}(123)\left(f_{2}(2)+f_{2}(1)\right) f_{4}(4)\left(f_{5}(5)+f_{5}(3)\right) \\
& =\mathfrak{F}(123) \hat{f}_{2}(2) \hat{f}_{4}(4) \hat{f}_{5}(5)
\end{aligned}
$$

Remark 4.4 If $L$ is the semilattice structure of the integers with respect to divisibility. By the specialization $\mathfrak{F}\left(\sigma_{3}, \ldots, \sigma_{k}\right)=\operatorname{sign}\left(\sigma_{3}\right) \ldots \operatorname{sign}\left(\sigma_{k}\right)$, one recovers the computations of Lehmer [G] as a special case of Theorem 3.3 and the result of Haukkanen ([19] Theorem 1. p 56) from Corollary 4.9.

\subsection{Factor closed subsets}

Let $S$ be a factor closed subset of $L$. Then, $f=\hat{f}$ and

$$
\operatorname{Det}_{\mathfrak{F}}\left(F_{x_{1}}\left(x_{1} \wedge \cdots \wedge x_{k}\right)\right)_{x_{1}, \ldots, x_{k} \in S}=\mathfrak{F}(\mathrm{Id}, \cdots, \mathrm{Id}) \prod_{x} f_{x}(x) .
$$


As special cases of equality (9), one recovers Lehmer's identities [9] and the original result of Smith [15].

\subsection{General case}

Let $X=\left\{x_{1}, \ldots, x_{n}\right\}$ be a subposet of a meet semi-lattice $L$. We will denote by $\bar{X}=\left\{x_{1}, \ldots, x_{n}, x_{n+1}, \ldots, x_{m}\right\}$ the smallest factor-closed subset of $L$ containing $X$. The aim of this section consists in investigating the $F$ determinant

$$
D F(X):=\operatorname{Det}_{\mathfrak{F}}\left(F_{x_{i_{1}}}\left(z_{x_{i_{1}}} \wedge x_{i_{2}} \wedge \cdots \wedge x_{i_{k}}\right)\right)_{1 \leq i_{1}, \ldots, i_{k} \leq n}
$$

where for each $x \in X, z_{x}$ denotes a fixed element of $\bar{X}$ such that $z \leq x$. As in the previous section, the result follows from the case $k=2$ and Proposition 2.1 .

Let us consider first the determinant $\operatorname{det}\left(F_{x_{i}}\left(z_{x_{i}} \wedge x_{j}\right)\right)_{1 \leq i, j \leq n}$. The functions $F_{x_{i}}$ can be chosen such that $F(x, y)$ is an incidence function. The set $\bar{X}$ being closed by factors, the functions $f_{x}$ and $\hat{f}_{x}$ are equal. Hence,

$$
F_{x_{i}}\left(z_{x_{i}} \wedge x_{j}\right)=\sum_{k=1}^{n+m} C_{x_{i}, x_{k}} \zeta\left(x_{k}, x_{j}\right)
$$

where

$$
C_{x, y}=f_{x}(y) \zeta\left(y, z_{x}\right)= \begin{cases}f_{x}(y) & \text { if } y \leq z_{x} \\ 0 & \text { otherwise }\end{cases}
$$

One has

\section{Proposition 4.5}

$$
\operatorname{det}\left(F_{x_{i}}\left(x_{i} \wedge x_{j}\right)\right)=\sum_{1 \leq k_{1}<\cdots<k_{n} \leq n+m} \operatorname{det}\left(C_{x_{i}, x_{k_{j}}}\right)_{1 \leq i, j \leq n} \operatorname{det}\left(\zeta\left(x_{k_{i}}, x_{j}\right)\right)_{1 \leq i, j \leq n} .
$$


Proof - By multi-linearity, one obtains

$$
\begin{aligned}
\operatorname{det}\left(F_{x_{i}}\left(x_{i} \wedge x_{j}\right)\right) & =\operatorname{det}\left(\sum_{k=1}^{n+m} C_{x_{i}, x_{k}} \zeta\left(x_{k}, x_{j}\right)\right) \\
& =\sum_{1 \leq k_{1}, \ldots, k_{n} \leq n+m} \operatorname{det}\left(C_{x_{i}, x_{k_{j}}} \zeta\left(x_{k_{j}}, x_{j}\right)\right) \\
& =\sum_{1 \leq k_{1}, \ldots, k_{n} \leq n+m} \operatorname{det}\left(C_{x_{i}, x_{k_{j}}}\right) \prod_{i} \zeta\left(x_{k_{i}}, x_{i}\right) \\
& =\sum_{1 \leq k_{1}<\cdots<k_{n} \leq n+m} \operatorname{det}\left(C_{x_{i}, x_{k_{j}}}\right)_{1 \leq i, j \leq n} \operatorname{det}\left(\zeta\left(x_{k_{i}}, x_{j}\right)\right)_{1 \leq i, j \leq n}
\end{aligned}
$$

Example 4.6 Let us consider the semilattice $L$ whose Hasse diagram is

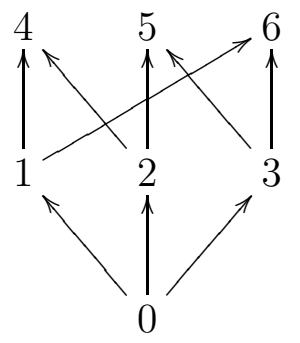

and $X=\{4,5,6\}$. We set $z_{4}=1, z_{5}=2$ and $z_{6}=6$. Consider the determinant

$$
\begin{aligned}
\operatorname{det}\left(F_{i}\left(z_{i} \wedge j \wedge k\right)\right)_{i, j, k \in X}= & \left|\begin{array}{ccc}
f_{4}(1)+f_{4}(0) & f_{4}(0) & f_{4}(1)+f_{4}(0) \\
f_{5}(2)+f_{5}(0) & f_{5}(2)+f_{5}(0) & f_{5}(0) \\
f_{6}(1)+f_{6}(0) & f_{6}(3)+f_{6}(0) & f_{6}(6)+f_{6}(3)+f_{6}(1)+f_{6}(0)
\end{array}\right| \\
= & -f_{4}(0) f_{6}(1) f_{5}(2)+f_{4}(1) f_{60} f_{5}(2)+f_{4}(1) f_{5}(0) f_{6}(3) \\
& +f_{41} f_{5}(0) f_{6}(6)+f_{4}(0) f_{5}(2) f_{63}+2 f_{4}(1) f_{5}(2) f_{6}(3) \\
& +f_{4}(1) f_{5}(2) f_{6}(6)
\end{aligned}
$$

Using the multilinearity of det, one recovers the expression given by Propo- 
sition $\longdiv { 4 . 5 }$

$$
\begin{aligned}
& \operatorname{det}\left(F_{i}\left(z_{i} \wedge j\right)\right)=\left|\begin{array}{ccc}
f_{4}(0) & f_{4}(1) & 0 \\
f_{5}(0) & 0 & f_{5}(2) \\
f_{6}(0) & f_{6}(1) & 0
\end{array}\right| \cdot\left|\begin{array}{ccc}
1 & 1 & 0 \\
1 & 0 & 1 \\
1 & 1 & 0
\end{array}\right|+\left|\begin{array}{ccc}
f_{4}(0) & f_{4}(1) & 0 \\
f_{5}(0) & 0 & 0 \\
f_{6}(0) & f_{6}(1) & f_{6}(3)
\end{array}\right| \cdot\left|\begin{array}{ccc}
1 & 1 & 0 \\
1 & 0 & 0 \\
1 & 1 & 1
\end{array}\right| \\
& +\left|\begin{array}{ccc}
f_{4}(0) & f_{4}(1) & 0 \\
f_{5}(0) & 0 & 0 \\
f_{6}(0) & f_{6}(1) & f_{6}(6)
\end{array}\right| \cdot\left|\begin{array}{lll}
1 & 1 & 0 \\
1 & 0 & 0 \\
1 & 1 & 1
\end{array}\right|+\left|\begin{array}{ccc}
f_{4}(0) & 0 & 0 \\
f_{5}(0) & f_{5}(2) & 0 \\
f_{6}(0) & 0 & f_{6}(3)
\end{array}\right| \cdot\left|\begin{array}{ccc}
1 & 0 & 0 \\
1 & 1 & 0 \\
1 & 0 & 1
\end{array}\right| \\
& +\left|\begin{array}{ccc}
f_{4}(1) & 0 & 0 \\
0 & f_{5}(2) & 0 \\
f_{6}(1) & 0 & f_{6}(3)
\end{array}\right| \cdot\left|\begin{array}{lll}
1 & 0 & 0 \\
0 & 1 & 0 \\
1 & 0 & 1
\end{array}\right|+\left|\begin{array}{ccc}
f_{4}(1) & 0 & 0 \\
0 & f_{5}(2) & 0 \\
f_{6}(1) & 0 & f_{6}(6)
\end{array}\right| \cdot\left|\begin{array}{lll}
1 & 0 & 0 \\
0 & 1 & 0 \\
1 & 0 & 1
\end{array}\right|
\end{aligned}
$$

More generally, one has a multi-indexed version of Proposition 4.5.

\section{Theorem 4.7}

$$
\begin{array}{r}
D F(X)=\sum_{1 \leq k_{1}<\cdots<k_{n} \leq n+m} \operatorname{Det}_{\mathfrak{F}}\left(f_{x_{i_{1}}}\left(x_{k_{i_{2}}}\right) \zeta\left(x_{k_{i_{2}}}, z_{x_{i_{1}}} \wedge x_{i_{3}} \wedge \cdots \wedge x_{i_{k}}\right)\right) \times \\
\times \operatorname{det}\left(\zeta\left(x_{k_{i}}, x_{j}\right)\right)
\end{array}
$$

Proof - We use Lemma 2.1 to expand $D F(X)$ and we obtain

$$
D F(X)=\sum_{\sigma_{1}, \ldots, \sigma_{k}} \mathfrak{F}\left(\sigma_{1}, \ldots, \sigma_{k}\right) \operatorname{det}\left(F_{x_{i}}\left(z_{x_{i}} \wedge x_{j} \wedge x_{\sigma_{3}(i)} \wedge \cdots \wedge x_{\sigma_{k}(i)}\right)\right) .
$$

Now, by Proposition 4.5, one gets

$$
\begin{aligned}
& D F(X)=\sum_{\sigma_{1}, \ldots, \sigma_{k}} \mathfrak{F}\left(\sigma_{1}, \ldots, \sigma_{k}\right) \sum_{1 \leq k_{1}<\cdots<k_{n} \leq n+m} \operatorname{det}\left(\zeta\left(x_{k_{i}}, x_{j}\right)\right) \times \\
& =\sum_{1 \leq k_{1}<\cdots<k_{n} \leq n+m}^{\times \operatorname{det}\left(f_{x_{i}}\left(x_{k_{j}} \zeta\left(x_{k_{j}}, z_{x_{i}} \wedge x_{\sigma_{3}(i)} \wedge \cdots \wedge x_{\sigma_{k}(i)}\right)\right)\right)} \operatorname{det}\left(\zeta\left(x_{k_{i}}, x_{j}\right)\right) \sum_{\sigma_{1}, \ldots, \sigma_{k}} \mathfrak{F}\left(\sigma_{1}, \ldots, \sigma_{k}\right) \times \\
& \times \operatorname{det}\left(f_{x_{i}}\left(x_{k_{j}} \zeta\left(x_{k_{j}}, z_{x_{i}} \wedge x_{\sigma_{3}(i)} \wedge \cdots \wedge x_{\sigma_{k}(i)}\right)\right)\right) \\
& \begin{aligned}
&= \sum_{\substack{1 \leq k_{1}<\cdots<k_{n} \leq n+m\\
\\
}} \times \operatorname{det}\left(\zeta\left(x_{k_{i}}, x_{j}\right)\right) \\
& \operatorname{Det}_{\mathfrak{F}}\left(f_{x_{i_{1}}}\left(x_{k_{i_{2}}}\right) \zeta\left(x_{k_{i_{2}}}, z_{x_{i_{1}}} \wedge x_{i_{3}} \wedge \cdots \wedge x_{i_{k}}\right)\right) \times \\
&
\end{aligned}
\end{aligned}
$$

This ends the proof.

Let set $F_{x_{1}}=F_{x_{2}}=\cdots=F_{x_{n}}=F$ then for $1 \leq k_{1}, k_{2}, \ldots, k_{n} \leq n$, we get 


\section{Corollary 4.8}

$$
\begin{array}{r}
\left.\operatorname{Det}_{\mathfrak{F}}\left(F\left(z_{x_{i_{1}}} \wedge x_{i_{2}} \wedge \cdots \wedge x_{i_{k}}\right)\right)=\sum_{\substack{1 \leq k_{1}<\cdots<k_{n} \leq n+m \\
\operatorname{Det}_{\mathfrak{F}}}} \prod_{i} f\left(x_{k_{i_{2}}}, z_{x_{i_{1}}} \wedge x_{i_{3}} \wedge \cdots \wedge x_{i_{i}}\right)\right) \operatorname{det}\left(\zeta\left(x_{k_{i}}, x_{j}\right)\right)
\end{array}
$$

Proof - By applying the equality,

$$
\begin{aligned}
& \operatorname{Det}_{\mathfrak{F}}\left(f\left(x_{k_{i_{1}}}\right) \zeta\left(x_{k_{i_{2}}}, z_{x_{i_{1}}} \wedge x_{i_{3}} \wedge \cdots \wedge x_{i_{k}}\right)\right)= \\
& \sum_{\sigma_{3}, \ldots, \sigma_{k}} \mathfrak{F}\left(\sigma_{3}, \cdots, \sigma_{k}\right) \operatorname{det}\left(f\left(x_{k_{i}}\right) \zeta\left(x_{k_{j}}, z_{x_{i}} \wedge x_{\sigma_{1}(i)} \wedge \cdots \wedge x_{\sigma_{k}(i)}\right)\right)= \\
& \prod_{i} f\left(x_{k_{i}}\right) \operatorname{Det}_{\mathfrak{F}}\left(\zeta\left(x_{k_{i_{2}}}, z_{x_{i_{1}}} \wedge x_{i_{3}} \wedge \cdots \wedge x_{i_{k}}\right)\right) .
\end{aligned}
$$

to identity 14, one obtains the result.

Remark 4.9 Assume that $L$ is the integer lattice. Then, if $z_{x}=x$ for each $x \in X$ and $\mathfrak{F}\left(\sigma_{3}, \cdots, \sigma_{k}\right)=\operatorname{sign}\left(\sigma_{3}\right) \ldots \operatorname{sign}\left(\sigma_{k}\right)$ in Corollary 4.8 , one recovers Theorem 2 in 19$]$. Moreover, Proposition 4.5 generalizes the result of $\mathrm{Li}[1 \mathrm{C}]$.

\section{References}

[1] T. Apostol, Introduction to Analytic Number Theory, Springer (1976)

[2] E. Altinisik, B.E. Sagan, N. Tuglu, GCD matrices, posets and nonintersecting paths, Linear and Multilinear Algebra 53(2) (2005) 75-84.

[3] S. Beslin and S. Ligh, Another generalization of Smith's determinant, Bull. Austral. Math. Soc, 40: 413-415 (1989)

[4] A Cayley, On the theory of determinants, Trans. Cambridge Phil. Soc. VIII (1843), 1-16.

[5] A Cayley, Mémoire sur les hyperdéterminants, Journ. Reine und Angew. Math., 30 (1846), 1-37.

[6] A Cayley, On the theory of permutants, Cambridge and Dublin Math. Journal VII (1851), 40-51. 
[7] A. Lascoux, Addition of 1, Séminaire Lotharingien, Mars 04. 8p.

[8] A. Lascoux, Multiplicative functions, http://www . combinatorics.net/lascoux/courses/dvi_ps/Moebiusps.rar

[9] D. H. Lehmer, The $p$ dimensional analogue of Smith's determinant, Amer. Math. Monthly 37:294-296 (1930).

[10] Z. Li, The determinants of gcd matrices, Linear Algebra Appl., 134:137$143(1990)$

[11] B. Lindström, Determinants on semilattices, Proc. Amer. Math. Soc. 20 (1969), 207-208.

[12] P. Haukkanen, Higher-Dimensionnal GCD matrices, Linear Algebra Appl., 170:53-63 (1992)

[13] C. Krattenthaler, Advanced determinant calculus: a complement, Linear Algebra Appl., 411 (2005), 68-166.

[14] L.H. Rice, P-way determinants with an application to transvectants, Amer. J. Math. 40:242-262 (1918)

[15] H. J. S. Smith, On the value of certain arithmetical determinant, Proc. London Math. Soc. (1) 7 (1876), 208-212 (p. 62)

[16] N P Sokolov, Spatial matrices and their applications (in Russian), Gosudarstv. Izdat. Fiz.-Mat. Lit., Moscow, 1960.

[17] N P Sokolov, Introduction to the theory of multidimensional matrices (in Russian), Nukova Dumka, Kiev, 1972. 